\title{
Control-system synthesis for open-loop unstable process with time delay
}

\author{
H.-P.Huang
}

C.-C.Chen

Indexing terms: Open-loop unstable poles, Control-system synthesis, Time delay

\begin{abstract}
To avoid performance limitations caused by an open-loop unstable pole, a threeelement structure, which is equivalent to a twodegrees-of-freedom control design, is proposed to synthesise a control system for an open-loop unstable process with time delay. Through this proposed approach, control problems such as stabilising an unstable pole, servo-tracking and disturbance rejection can be treated independently. The resulting three-element structure can then be used to derive conventional two-degrees-of-freedom elements or conventional PID systems. Tuning rules for a PID controller are also provided. Examples are presented to illustrate the proposed method.
\end{abstract}

\section{Introduction}

Control system design for an open-loop unstable process is more difficult than that for a stable one. The difficulties are mostly due to the unstable nature of the dynamics, for which most design tools cannot be used. For example, the Bode stability criterion and the pole/ zero cancellation method become inapplicable when an unstable pole exists [1]. The strongly stabilising problem is also an immense obstacle for the unstable process [2]. The parity-interlacing property makes it clear that there are times when right-halfplane poles must be added in the compensator in order to stabilise the unstable process. Except for the stability problems, the performance specification which can be assigned to the control system for an unstable process is also different from that for a stable one. Some performance specifications which are very common for stable processes, however, would be impossible to achieve for unstable processes.

The performance limitations due to the right-halfplane (RHP) poles and zeros in the process are discussed very early by Youla, Bongiorno and Jabr [3]. They pointed out that every RHP pole of the process is a zero of the sensitivity function of at least the same multiplicity. Freudenberg and Looze made a series of

\section{(C) IEE, 1997}

IEE Proceedings online no. 19971222

Paper first received 8th July 1996 and in revised form 7th March 1997

The authors are with the Department of Chemical Engineering, National Taiwan University, Taipei 10617, Taiwan, Republic of China studies on performance limitations relating to RHP poles and zeros in frequency domain [4 6]. Their work showed that these performance limitations are not only imposed on a specific frequency but also interfere with each other at all frequencies. These effects are important to the loop-shaping design method [7]. In this paper, such limitations in the frequency domain are found to be a lower bound of the complementary sensitivity function. Moreover, performance limitations in the time domain for time-delayed unstable processes are also discussed. These problems of performance limitations are discussed in Section 2.

The objectives for controlling an open-loop unstable process should include: stabilising the unstable pole, achieving good performance in servo-tracking and in disturbance rejection. As is discussed in Section 2, the existing design methods, including those of the 2degree-of-freedom (2DF) control system, are, as usual, inadequate to achieve all these three objectives satisfactorily. Consequently, the success of a design is very much dependent on a case-by-case basis. To make up for this deficiency, a design method for the control of time-delayed unstable processes, in which the stability problem and performance problem can be taken into account separately, is thus proposed. The proposed method is conducted in a three-element structure which has three controllers. Each of these controllers has a different purpose, as mentioned above. After completion of these controllers in the three-element structure, the controllers in the conventional 2DF system are then directly derived from the three-element structure. In Section 3, the problems of designing these three controllers in the three-element structure and the equivalence between the three-element structure and conventional 2DF structure are discussed.

Since the PID controller has been considered to be the most widely used controller in industry, a PID controller tuning method evolved from the three-element structure is proposed in the paper.

Although tuning of PID controllers for time-delayed unstable processes has been an active area of research in the literature, most work has dealt only with firstorder time-delayed unstable processes. Moreover, all these studies only apply to the case where the ratio of delay to unstable time constant (i.e. $\theta / T$ ) is smaller than unity [8-13]. In this paper, the proposed PID method can apply to the case where $\theta / T$ is smaller than 2 for the first-order time-delayed unstable process, and is not limited to first-order unstable processes only. All the problems in tuning the PID controllers are discussed in Section 4. 
In Section 5, three examples are simulated to show the performance and robustness of the proposed method. These examples include two first-order examples with $\theta / T<1$ and $\theta / T>1$, respectively. The third example is an example of a high-order process.

\section{Performance limitations}

The existence of the RHP poles or RHP zeros in an open-loop transfer function of a process will cause some inevitable limitations on the performance of closed-loop control. Traditionally, these limitations are expressed in terms of the sensitivity function or the complementary sensitivity function [3]. Freudenberg and Looze pointed out that the existence of the RHP poles and RHP zeros would result in interactions in all frequencies [4-6]. The Bode integral relation was then extended to include processes having unstable poles in their works. In this paper, it is shown that such an integral relation will result in a lower bound of the complementary sensitivity function. Performance limitations in the time domain for time-delayed unstable processes are also discussed.

\subsection{Time-domain limitation}

If the process has an open-loop unstable pole, the response of the closed-loop system would inevitably go higher than the reference value in all cases. This fact can be easily understood from the following theorem.

\section{Theorem 1}

Consider the unity-feedback control structure (Fig. 1). Assume that there is a RHP pole $\lambda$ in the process, that the corresponding feedback system is stable, and that the input signal $v(t)$ is also stable. Then the error signal $e(t)$ must satisfy the integral constraint

$$
\int_{0}^{\infty} \mathrm{e}^{-\lambda t} e(t) d t=0
$$

Proof

$$
\begin{aligned}
\int_{0}^{\infty} \mathrm{e}^{-\lambda t} e(t) d t & =\left.E(s)\right|_{s=\lambda} \\
& =\left.S(s) v(s)\right|_{s=\lambda} \\
& =\left.\frac{1}{1+L(s)} v(s)\right|_{s=\lambda} \\
& =\frac{v(\lambda)}{1+L(\lambda)} \\
& =0
\end{aligned}
$$

where: $S(s)$ is the sensitivity function, $v(s)$ is the Lapalace transform of the input signal, $E(s)$ is the Lapalace transform of the error signal, and $L(s)$ is the loop transfer function.

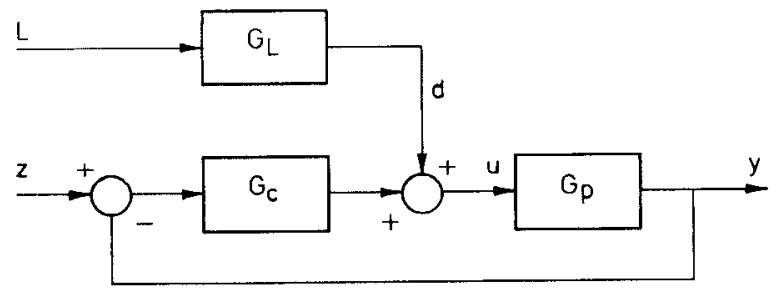

Fig. 1 Unity-feedback structure

Theorem 1 shows that, if the RHP pole of the process is a real number, then any negative error in the time domain should be compensated by the positive errors.
For a step change in setpoint, the output of the process at the start time will fall behind the new setpoint value; therefore, the system will result in a negative error in the start. Consequently, the output of the process should rise higher than the new setpoint value to compensate these starting errors.

In addition to the RHP poles, time delay in the process will also cause performance degradation in the control system. In the following, it is shown that the maximum value of the process output would be bounded from below due to the RHP pole and time delay.

\section{Corollary 2}

Consider the unity-feedback control structure. Assume that there is a real RHP pole $\lambda$ in the process, that the corresponding feedback system is stable, and that the input signal is a unity step change in setpoint $r(t)$. In addition, assume that there is a time delay $\theta$ in the process. Then, the maximum value of the output $y_{\max }$ will be bounded from below:

$$
y_{\max } \geq \mathrm{e}^{\lambda \theta}
$$

\section{Proof}

From eqn. 1, and the following relations:

$$
\begin{aligned}
& r(t)=1, \forall t \\
& y(t)=0, t \in[0, \theta] \\
& e(t)=1, t \in[0, \theta] \\
& e(t)=r(t)-y(t)>1-y_{\max }, \forall t
\end{aligned}
$$

we have:

$$
\begin{aligned}
& -\int_{\theta}^{\infty} \mathrm{e}^{-\lambda t} \mathrm{e}(t) d t=\int_{0}^{\theta} \mathrm{e}^{-\lambda t} d t \\
& \left(y_{\max }-1\right) \int_{\theta}^{\infty} \mathrm{e}^{-\lambda t} d t \geq \int_{0}^{\theta} \mathrm{e}^{-\lambda t} d t \\
& \left(y_{\max }-1\right) \frac{\mathrm{e}^{-\lambda \theta}}{\lambda} \geq \frac{-1}{\lambda}\left(\mathrm{e}^{-\lambda \theta}-1\right) \\
& y_{\max } \geq \mathrm{e}^{\lambda \theta}
\end{aligned}
$$

Corollary 2 shows that, if the control system for a time-delayed unstable process produces no steady-state error, then the output of the process will have at least an overshoot of $e^{\lambda \theta}-1$. For controlling stable processes, the overshoot of the output can be suppressed arbitrarily to a desired value, for example less than $15 \%$ as usual [14]. However, as shown by Corollary 2, the magnitude of overshoot cannot be assigned arbitrarily for a time-delayed unstable process.

The ability of a control system to reject disturbances will also degrade if there are RHP poles in the process. To avoid disturbance rejection, the output of the process should be kept as close to the setpoint as possible, but the minimum value of the process output is bounded from above if there are unstable poles and time delay in the process.

\section{Corollary 3}

Consider the unity-feedback control structure. Assume that there is a real RHP pole $\lambda$ in the process, that the corresponding feedback system is stable, and that the input signal is a unity step disturbance $d(t)$ at the output of the process. In addition, assume that there is a time delay $\theta$ in the process. Then, the minimum value of the output $y_{\min }$ will be bounded from above:

$$
y_{\min } \leq 1-\mathrm{e}^{\lambda \theta}
$$


where

$$
y_{\min } \triangleq \inf _{t}\{y(t)\}=-\sup _{t}\{-y(t)\}
$$

Proof

We have $y(t)=d(t)=1,0<t<\theta$, and the following equation follows eqn. 1 immediately:

$$
\begin{aligned}
& \int_{0}^{\theta} \mathrm{e}^{-\lambda t}(-1) d t+\int_{\theta}^{\infty} \mathrm{e}^{-\lambda t}\{-y(t)\} d t=0 \\
& \int_{\theta}^{\infty} \mathrm{e}^{-\lambda t} y(t) d t=-\int_{0}^{\theta} \mathrm{e}^{-\lambda t} d t \\
& y_{\min } \int_{\theta}^{\infty} \mathrm{e}^{-\lambda t} d t \leq-\int_{0}^{\theta} \mathrm{e}^{-\lambda t} d t \\
& y_{\min } \leq 1-\mathrm{e}^{\lambda \theta}
\end{aligned}
$$

Note that the bounds proposed in corollaries 2 and 3 are very conservative. It is understood from the fact that both proposed bounds are reachable only under the assumption that an infinitely large control action is possible. This control action can only be realised by infinitely high controller gain. However, the maximum feedback gain is always limited in a time-delayed unstable process. Although the proposed bounds seems too conservative to be useful, corollaries 2 and 3 still provide us important implications on performance problems for controlling time-delayed unstable processes. First, some impossible performance specifications about overshoot can be ruled out. Secondly, as these corollaries show, in general, the performance of a control system will become worse as the product of $\lambda$ and $\theta$ increases.

\subsection{Frequency-domain limitation}

In the frequency domain, the maximum magnitude of the complementary sensitivity function of a control system $T(j \omega)$ is an important index for both the control performance and stability robustness. Here it is shown that the $\infty$-norm of such a $T(j \omega)$ has a lower bound due to the presence of the RHP pole and time delay. To do this, the following definition and theorem proposed by Freudenberg and Looze are referred to first [4].

\section{Definition}

The Blaschke product for RHP zeros $B_{z}(s)$ is defined as:

$$
B_{z}(s) \triangleq \prod_{i=1}^{N_{z}} \frac{-s+z_{i}}{s+z_{i}^{H}}
$$

where $z_{i}$ is the open-loop RHP pole, and $z_{i}^{H}$ is the conjugate of $z_{i}$.

Theorem 4 (Freudenberg and Looze, 1985 [4])

Let $\lambda=x+i y$ be a RHP pole of the plant $G_{p}(s)$; then the complementary sensitivity function $T(s)$, will satisfy

$$
\int_{-\pi / 2(\infty)}^{\pi / 2(\infty)} \ln |T(j \omega)| d \theta_{\lambda}(\omega)=\pi \ln \left|B_{z}^{-1}(\lambda)\right|+\pi x \theta
$$

where $\theta_{\lambda}(\omega)=\tan ^{-1}(\omega-y) / x$

As a complement to theorem 4 , the following corollary is proposed to emphasise the lower bound of the complementary sensitivity function caused by delay and the RHP pole in the process:

\section{Corollary 5}

If a plant $G_{p}(s)$ has a time delay $\theta$ and a RHP pole $\lambda=$ $x+i y$, then the complementary sensitive function $T(s)$, will satisfy the relation

where

$$
\|T(\cdot)\|_{\infty} \geq e^{x \theta}
$$

$$
\|T(\cdot)\|_{\infty} \equiv \sup _{\omega}|T(j \omega)|
$$

Proof

It is obvious that

By definition,

$$
\int_{-\pi / 2(\infty)}^{\pi / 2(\infty)} d \theta_{\lambda}(\omega)=\pi
$$

$$
\begin{aligned}
& B_{z}(s)=\prod_{i=1}^{N_{z}} \frac{-s+z_{i}}{s+z_{i}^{H}} \\
& \rightarrow\left|B_{z}(\lambda)\right| \leq 1 \quad \text { for } \lambda=x+i y, x \geq 0 \\
& \rightarrow\left|B_{z}^{-1}(\lambda)\right| \geq 1 \\
& \rightarrow \ln \left|B_{z}^{-1}(\lambda)\right| \geq 0
\end{aligned}
$$

Substituting this result into theorem 4 ,

$$
\begin{aligned}
& \int_{-\pi / 2(\infty)}^{\pi / 2(\infty)} \ln |T(j \omega)| d \theta_{\lambda}(\omega) \geq \pi x \theta \\
& \ln \|T(\cdot)\|_{\infty} \int_{-\pi / 2(\infty)}^{\pi / 2(\infty)} d \theta_{\lambda}(\omega) \geq \pi x \theta \\
& \ln \|T(\cdot)\|_{\infty} \geq x \theta \\
& \|T(\cdot)\|_{\infty} \geq \mathrm{e}^{x \theta}
\end{aligned}
$$

The ratio of $\|\left. T(\cdot)\right|_{\infty}$ to $\|T(0)\|$ is referred to as the peak gain ratio in most control textbooks $[14,1]$. For stable processes, the peak gain ratio of the control system is usually assigned to be about 1.3 [15]. However, as eqn. 4 shows, it is impossible to require the peak gain ratio to be about 1.3 for some time-delayed unstable processes.

\section{Control-system synthesis in three-element structure}

Although there are many studies which discuss the the issue of tuning a PID controller for a time-delayed unstable process in the unity-feedback structure [8-13], however, as shown in the preceding section, the performance improvement of the control system is limited if a unity-feedback structure is considered. Thus, the 2DF control structure should be considered to improve the performance. Nevertheless, studies on 2DF structure are still rare [16-18].

In Quinn and Sanathanans' work, the delay is approximated to a rational function by using the second-order Pade approximation [16]. Then, a method similar to the linear algebraic method [19] is introduced to design the controllers based on the approximate model. The disadvantages of such a method are: First, it is necessary to assign a reference model of the overall system, but it is still unclear how such a reference model can be chosen which satisfies a specific performance specification for unstable processes. Moreover, the zeros of such a reference model cannot be arbitrarily assigned; thus, trial-and-error steps are needed to find an acceptable reference model. Secondly, as pointed out in the literature, such a method is applicable only for a lower ratio of time delay to unstable time constant. For larger values of such a ratio, the method is reported not to be robust for perturbation in parameters [18]. 
Internal model control (IMC) is a powerful method for control-system synthesis, and it is well known that the control system cannot be implemented by the IMC structure if the process is an unstable one [17]. However, as suggested by Morari and Zafiriou, one can still design the controllers using the IMC method, and then, implement the controllers in an equivalent-feedback structure. This suggestion is only true for processes without delay, but would encounter difficulties for time-delayed unstable processes. The following example illustrates these difficulties. Suppose the input signal $v$ $=1 / \mathrm{s}$ and the process considered is

$$
P(s)=\frac{\mathrm{e}^{-\theta s}}{-s+\beta}
$$

The optimal $\mathrm{H}_{2}$ IMC controller is

$$
\tilde{q}(s)=\frac{(-s+\beta)\left\{\left(2 \mathrm{e}^{\theta \beta}-1\right) s+\beta\right\}}{s+\beta}
$$

The standard IMC filter is chosen to meet the requirements of internal stability and pole zero excess:

$$
f(s)=\frac{\left(\tau_{f}^{2} \beta+2 \tau_{f}\right) s+1}{\left(\tau_{f}^{2} s+1\right)^{2}}
$$

So, the IMC controller is

$$
\begin{aligned}
q(s) & =\tilde{q}(s) f(s) \\
& =\frac{(-s+\beta)\left\{\left(2 \mathrm{e}^{\theta \beta}-1\right) s+\beta\right\}\left\{\left(\tau_{f}^{2} \beta+2 \tau_{f}\right) s+1\right\}}{(s+\beta)\left(\tau_{f} s+1\right)^{2}}
\end{aligned}
$$

The equivalent feedback controller is derived from $C(s)$ $=q(s) /\{1-q(s) P(s)\}$, so we have

$$
C(s)=\frac{(-s+\beta)\left\{\left(2 \mathrm{e}^{\theta \beta}-1\right) s+\beta\right\}\left\{\left(\tau_{f}^{2} \beta+2 \tau_{f}\right) s+1\right\}}{(s+\beta)\left(\tau_{f} s+1\right)^{2}-\left\{\left(2 \mathrm{e}^{\theta \beta}-1\right) s+\beta\right\}\left\{\left(\tau_{f}^{2} \beta+2 \tau_{f}\right) s+1\right\} e^{-\theta s}}
$$

After a detailed inspection on $C(s)$, it is found that $s=$ $\beta$ is both the zero and pole of $C(s)$. Because the denominator of $C(s)$ is not in a rational form, obviously, it is not possible to cancel $s=\beta$ in $C(s)$ explicitly. Although a controller of the form $G_{c}(s)=X(s) /\left\{Y(s)-Z(s) e^{-\theta s}\right\}$ can be implemented by the structure shown in Fig. 2, however, owing to the presence of the unstable factor $s$ $=\beta$ in $X(s)$, it is not admissible to execute the IMC feedback controller by such a structure [3]. Thus, to implement the IMC feedback controller for a timedelayed unstable process, it is necessary to approximate $C(s)$ by some other method to cancel the unstable factor. Doing so unavoidably makes the design procedure very tedious. In addition to this implementation problem, another major disadvantage of using the IMC method directly is that the design of IMC filter for an unstable process is never as simple as for the stable case, and the robust-stability problem is not clearly related to the IMC filter constant as in the stable case [20].

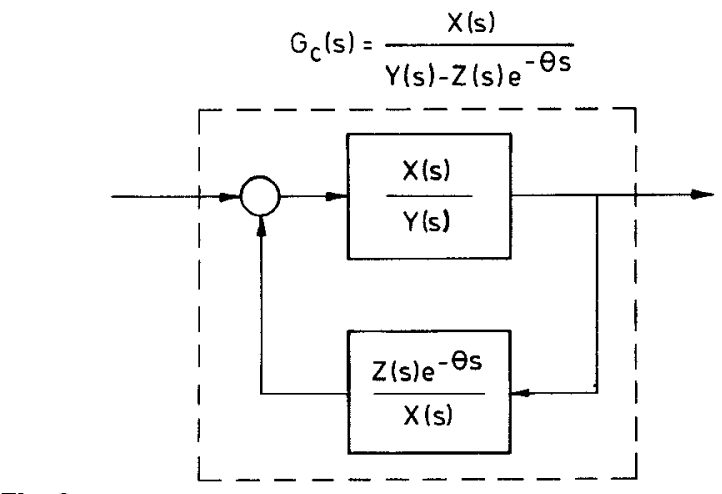

Fig. 2 Imlementation of $\bar{C}_{2}$ and $\bar{C}_{3}$
Recently, Jacob and Chidambaram discussed some strategies in the design of controllers for time-delayed unstable processes in the 2DF structure [18]. However, their work was limited to the first-order process only, and because the controller in their work is assumed to be a P/PI controller, these methods are applicable only for processes in which the ratio of delay to unstable time constant is less than unity.

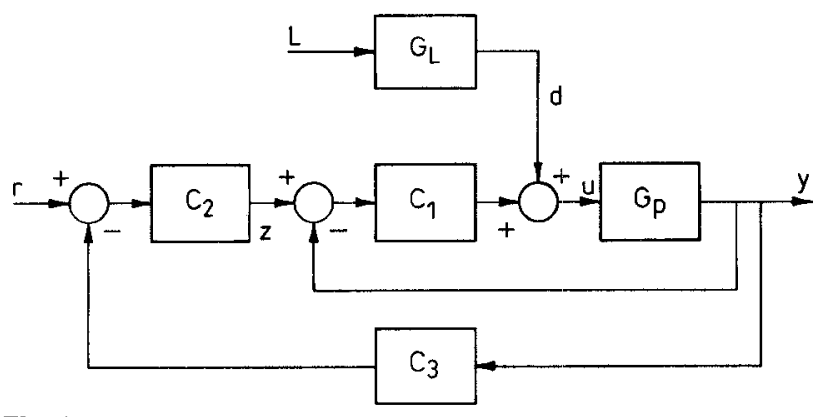

Fig.3 Three-element structure for unstable processes

As pointed out by many studies in the literature, to control a time-delayed unstable system is by no means a simple task [8-12]. Thus, direct design of controllers in a 2DF structure is difficult for time-delayed unstable processes, because it is hard to handle stability and performance problems simultaneously. To make the relation of the design procedures to the specification requirements more transparent, a three-element control structure, as shown in Fig. 3, is thus proposed to design a 2DF system. This structure has three controllers which are designed for different objectives. Of the three controllers, $C_{1}$ in the inner loop is provided to stabilise the unstable pole first. The other two controllers in the outer loop are then used to take care of servotracking and disturbance rejection by considering the inner loop as an open-loop stable process. Although there are three controllers in the proposed structure, this structure is, with one assumption which does not lose generality, equivalent to the 2-degree-of-freedom (2DF) feedback structure shown in Fig. 4. Thus, the proposed structure is used to handle the stability and performance problems independently in controller design steps, and it affords a target system from which the desired 2DF control system can be derived. This advantage makes the work of designing more straightforward and easier than designing in the conventional 2DF structure. This is discussed in detail below.

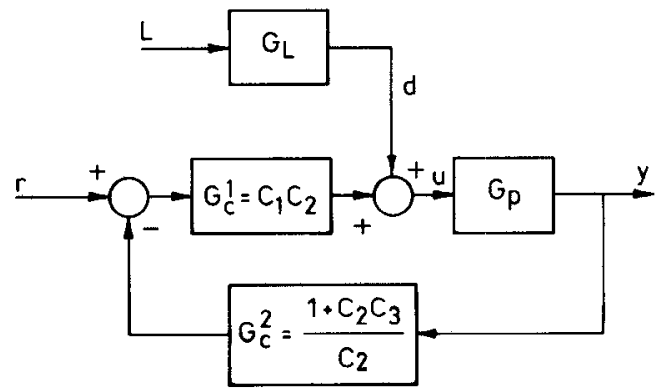

Fig.4 Conventional 2DF structure I

To illustrate the equivalence of the proposed structure and the 2DF structure shown in Fig. 4, refer to Fig. 3 first. It is straightforward to derive the following relation (where $d$ is assumed to be zero):

$$
u=C_{1} C_{2} r-C_{1}\left(1+C_{2} C_{3}\right) y
$$


Thus, using the structure in Fig. 3 as a target, the control system in Fig. 4, which has two controllers (i.e. $G_{c}{ }^{1}$ and $G_{c}^{2}$ ), is constructed as:

$$
\begin{gathered}
G_{c}^{1}=C_{1} C_{2} \\
G_{c}^{2}=\frac{\left(1+C_{2} C_{3}\right)}{C_{2}}
\end{gathered}
$$

Note that, unless $C_{2}$ is semiproper (biproper), the system in Fig. 3 will not be equivalent to that in Fig. 4 completely. However, this is usually true in practice, for example in PID controllers and lead-lag controllers. If $C_{2}$ is semiproper, the proposed structure can be implemented by the $2 \mathrm{DF}$ structure in Fig. 4 , and the feedback controllers $G_{c}^{1}$ and $G_{c}^{2}$ can be thus synthesised according to eqns. 6 and 7 .

\subsection{Design $C_{1}$ for stabilising general unstable processes}

The controller $C_{1}$ is provided to stabilise the plant $G_{p}$ so that the inner loop becomes stable. No performance issue is considered in this step for this stabilised inner loop. As the parity-interlacing property shows, we may fail to stabilise some unstable processes even without time delay, if only stable controllers are to be considered. Thus, only the following two types of timedelayed unstable processes are considered in this work:

$$
\begin{gathered}
G_{p}(s)=\frac{K_{p} \mathrm{e}^{-\theta s}}{T s-1} \\
G_{p}(s)=\frac{K_{p} \mathrm{e}^{-\theta s}}{(T s-1)(a s+1)}
\end{gathered}
$$

For convenience, these two processes are termed the FODUP (first-order delayed unstable process) and the SODUP (second-order delayed unstable process), respectively. It is well known that a higher-order stable part of a transfer function can be approximated effectively to a stable first-order transfer function with time delay. Thus, the proposed method still can apply to the higher-order processes.

It has been reported in the literature that a well tuned P/PI controller could stabilise a FODUP if and only if $\theta / T<1[21,11]$. A recent study shows that this constraint will relax to $\theta / T<2$ if the PD/PID controller is considered [22]. It was also known that a SODUP could be stabilised by a well tuned $\mathbf{P}$ controller if and only if $\theta / T+a / T<1$. Thus, if a PD controller is used to cancel the stable pole of a SODUP this constraint can be relaxed to $\theta / T<1$ for a SODUP.

The loop gain which stabilises a time delayed unstable process is always bounded from both below and above. The maximum loop gain $K_{M}$ and the minimum loop gain $K_{m}$ which stabilise the system can be calculated from the phase criterion of root loci. Consider the FODUP as an example. The intersections of root loci and the $j \omega$ axis in the first branch $(0 \leq \omega<\pi / \theta)$ are determined by the equation

$$
-\theta \omega-\pi+\tan ^{-1} T \omega=-\pi
$$

i.e.

$$
\theta \omega=\tan ^{-1} T \omega
$$

Obviously, $\omega=0$, which corresponds to $K_{m}$, is one solution of eqn. 10. It was reported that there is one solution other than $\omega=0$, if the value of $\theta / T$ is smaller than unity $[11,23]$. The loop gain corresponding to those intersections is thus determined by

$$
K=\sqrt{1+T^{2} \omega^{2}}
$$

Numerical values of $K_{m}$ and $K_{M}$ for the FODUPs corresponding to different value of $\theta / T(\triangleq \hat{\theta})$ are calculated out and plotted in Fig. 5. As shown in that Figure, the value of $K_{m}$ is independent of $\theta / T$, and the value of $K_{M}$ is a monotonically decreasing function of $\theta / T$.

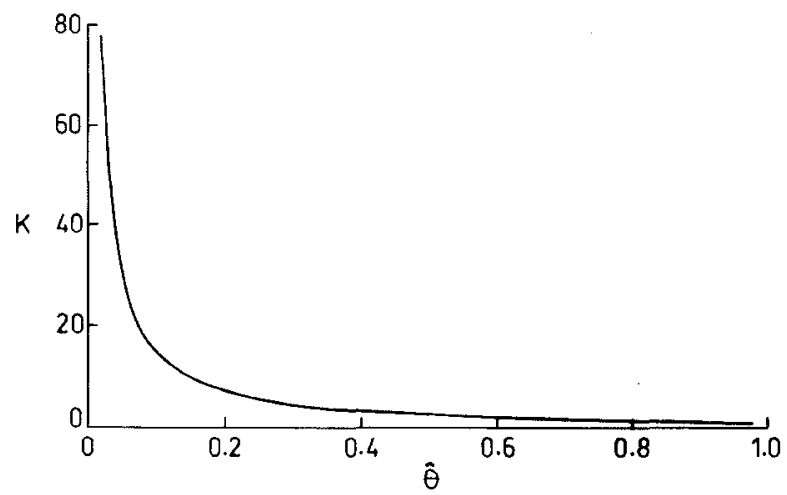

Fig. $5 K_{m}$ and $K_{M}$ of FODUP

$G_{p}(s)=\exp (-\theta s) /\left(T_{s}-1\right)$

$\stackrel{K_{m}}{\cdots \cdots \cdots . . . .} K_{m}$

In this paper, $C_{1}$ is chosen to be a PD controller [i.e. $\left.G_{c}(s)=K_{c}(b s+1)\right]$. Such a choice makes the proposed method applicable to $\theta / T<2$ for a FODUP. As shown in the work of Rotstein and Lewin [9], the loop gain is an important parameter to handle the robustness issues of the control system; thus, it seems interesting to see which value of $b / T(\triangleq b)$ could make the feasible interval of loop gain $\left(K_{m}, K_{M}\right)$ as large as possible. For the FODUPs, the optimal value of $\hat{b}$ in this sense is solved for different values of $\theta / T$, and the results are plotted in Fig. 6.

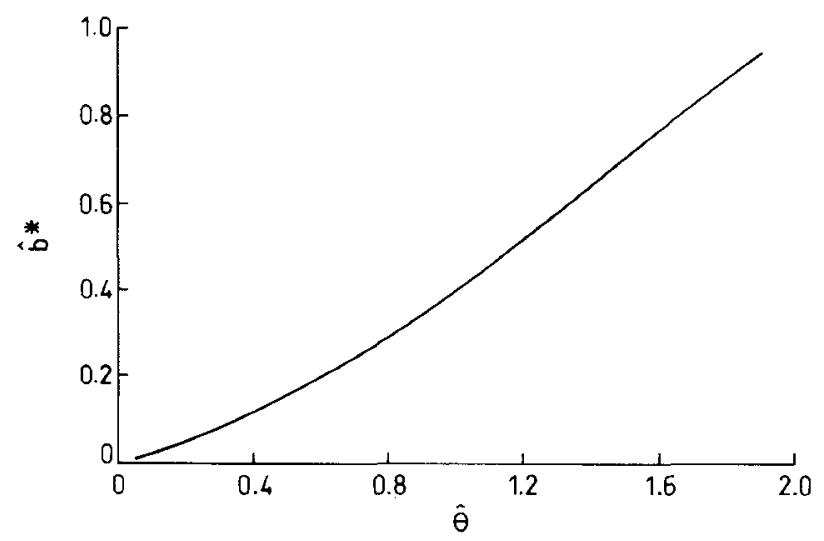

Fig.6 Optimal b for FODUP

In this work, $C_{1}$ is aimed at stabilising the problem; thus, $C_{1}$ is constructed as follows.

Case (i): FODUP

The value of $\bar{b}$ is chosen to make the feasible interval of $\left(K_{m}, K_{M}\right)$ largest, and the controller gain $K_{c}$ is chosen such that the loop gain (i.e. $\left.K_{c} K_{p}\right)$ equals to $\frac{1}{2}\left(K_{m}+\right.$ $\left.K_{M}\right)$.

Case (ii): SODUP

The value of $b$ is chosen to cancel the stable pole (i.e. $b$ $=a$ ), and the controller gain $K_{c}$ is chosen such that the loop gain (i.e. $\left.K_{c} K_{p}\right)$ equals to $\frac{1}{2}\left(K_{m}+K_{M}\right)$.

In foregoing cases, the loop gain is chosen to be the mean value of $K_{m}$ and $K_{M}$. Such a choice is due to the 
robustness-stability consideration. For other purposes, the loop gain may be chosen by another method, for example, the optimum-gain-margin method suggested by De Paor and O'Malley [8]. The issues of robustness stability about the inner loop of the proposed three-element structure are discussed in the following two theorems.

\section{Theorem 6}

Consider the open-loop transfer function

$$
G_{l p}=\frac{K \mathrm{e}^{-\theta s}}{T s-1}
$$

Suppose that the uncertainties occur only in $\theta$ and $T$, and that the closed-loop system is stable in the nominal case. Thus, the perturbed system is stable, if and only if

$$
\frac{\theta}{T}<\frac{\tan ^{-1} \sqrt{K^{2}-1}}{\sqrt{K^{2}-1}}
$$

\section{Proof}

It is reported in the literature that $K_{M}$ is a monotonically decreasing function of $\theta / T$, and $K_{m}$ is independent of $\theta / T[8,23]$. Furthermore, the closed-loop system is assumed to be stable in the nominal case, and this assumption implies $K>1$. So, the perturbed system is stable if and only if $K<K_{M}(\theta / T)$. In the critical case,

So

$$
\begin{aligned}
& K=K_{M}=\sqrt{1+T^{2} \omega^{2}} \\
& \theta \omega=\tan ^{-1} T \omega
\end{aligned}
$$

$$
\frac{\theta}{T}=\frac{1}{T \omega} \tan ^{-1} T \omega=\frac{\tan ^{-1} \sqrt{K^{2}-1}}{\sqrt{K^{2}-1}}
$$

Recalling that $K_{M}$ is a monotonically decreasing function of $\theta / T$ thus $K<K_{M}$ if and only if

$$
\frac{\theta}{T}<\frac{\tan ^{-1} \sqrt{K^{2}}-1}{\sqrt{K^{2}-1}}
$$

\section{Theorem 7}

Consider the open-loop transfer function

$$
G_{l p}(s)=\frac{K(b s+1) \mathrm{e}^{-\theta s}}{T s-1}
$$

Suppose that the uncertainty occurs only in $\theta$, and that the closed-loop system is stable in the nominal case. Then, the closed-loop system is stable, if and only if

$$
\frac{\theta}{T}<\frac{\tan ^{-1} \sqrt{\frac{\left(K^{2}-1\right) T^{2}}{-K^{2} b^{2}+T^{2}}}+\tan ^{-1} \frac{b}{T} \sqrt{\frac{\left(K^{2}-1\right) T^{2}}{-K^{2} b^{2}+T^{2}}}}{\sqrt{\frac{\left(K^{2}-1\right) T^{2}}{-T^{2} b^{2}+T^{2}}}}
$$

Proof

The proof is trivially similar to that of theorem 6 .

With the aid of the foregoing two theorems, the allowable parameter variations in $\theta$ and $T$ can be estimated for the proposed method, if the uncertainty does not occur in $K_{p}$. If the uncertainty in $K_{p}$ occurs, theorems similar to the above two still can be derived, but the uncertainty bound on $K_{p}$ should be known first.

\subsection{Design of $\mathrm{C}_{2}$ and $\mathrm{C}_{3}$}

Referring to Fig. 3, the dynamic equation of the the inner loop is

$$
\begin{aligned}
y & =\frac{C_{1} G_{p}}{1-C_{1} G_{p}} z+\frac{G_{p}}{1+C_{1} G_{p}} d \\
& =\hat{G}_{p} z+\hat{G}_{d} d \\
& =\hat{G}_{p} z+\hat{G}_{L} L
\end{aligned}
$$

where $d=G_{L} L$
If $\hat{G}_{p}$ and $\hat{G}_{d}$, which are open-loop stable, are known, design of $C_{2}$ and $C_{3}$ for either servotracking or disturbance rejection becomes easy and straightforward. The dynamics of $\hat{G}_{p}$ and $\hat{G}_{L}\left(\triangleq G_{d} G_{L}\right)$ can be expressed either in nonparametric form or in parametric form. For the former, one can prepare the Bode plot of $\hat{G}_{p}$ and $\hat{G}_{L}$ and read the key parameters such as bandwidth, critical gains and critical frequency accordingly. The resulting parameters can then be used to design $C_{2}$ and $C_{3}$ for either purpose [19]. Alternatively, one can find the best approximate transfer functions of $\hat{G}_{p}$ and $\hat{G}_{L}$ using the parametric-optimisation method to fit in the Bode plot, and then design controllers according to these approximate models.

In this paper, controllers $C_{2}$ and $C_{3}$ are designed by employing the IMC method according to the stable transfer function $\hat{G}_{p}$ and $\hat{G}_{L}$. The whole design process is illustrated in the following.

Case (i): FODUP

$$
G_{p}(s)=\frac{K_{p} \mathrm{e}^{-\theta s}}{T s-1}
$$

Then the stabilising controller $C_{1}$ will be

$$
C_{1}(s)=K_{c}(b s+1)
$$

where $b$ and $K_{c}$ are constructed as discussed in Section 3.1. Such a design of controller $C_{1}$ will make the inner loop of Fig. 3 stable. In other words, the following equation has no RHP roots:

$$
T s-1+K(b s+1) \mathrm{e}^{-\theta s}=0
$$

where $K=K_{c} K_{p}$, so that

$$
\begin{gathered}
\hat{G}_{P}=\frac{K(b s+1) \mathrm{e}^{-\theta s}}{T s-1+K(b s+1) \mathrm{e}^{-\theta s}} \\
\hat{G}_{L}=\frac{K p \mathrm{e}^{-\theta s}}{T s-1+K(b s+1) \mathrm{e}^{-\theta s}} G_{L}(s)
\end{gathered}
$$

According to $\hat{G}_{P}$,

$$
\begin{aligned}
{\left[\hat{G}_{P}\right]_{-}=} & \frac{K(b s+1)}{T s-1+K(b s+1) \mathrm{e}^{-\theta s}} \\
& {\left[\hat{G}_{p}\right]_{+}=\mathrm{e}^{-\theta s} }
\end{aligned}
$$

The IMC controllers $q_{a}$ and $q_{b}$ (referring to Fig. 7) are defined as

$$
\begin{aligned}
& q_{a}=f_{a}\left[\left(\hat{G}_{p}\right)_{-}\right]^{-1} \\
& q_{b}=f_{b}\left[\left(\hat{G}_{p}\right)_{-}\right]^{-1}
\end{aligned}
$$

where $f_{a}$ and $f_{h}$ are two standard IMC filters which serve the two degrees of freedom to shape the system for servotracking and for disturbance rejection, respectively. Then controllers $C_{1}$ and $C_{2}$ are derived as

$$
\begin{gathered}
C_{2}=\frac{q_{a}}{1-q_{b} \hat{G}_{p}} \\
C_{3}=\frac{q_{b}}{q_{a}}
\end{gathered}
$$

The controllers $C_{2}$ and $C_{3}$ can be implemented by the structure shown in Fig. 2. The whole system can also be implemented in the stable IMC structure or in the $2 \mathrm{DF}$ structure. However, in the $2 \mathrm{DF}$ structure, two controllers (i.e. $G_{c}^{1}$ and $G_{c}^{2}$ ) are needed instead of three in the three-element structure (i.e. $C_{1}, C_{2}$ and $C_{3}$ ). 
Case (ii): SODUP

$$
G_{p}(s)=\frac{K_{p} \mathrm{e}^{-\theta s}}{(T s-1)(a s+1)}
$$

Then the stabilising controller $C_{1}$ will be

$$
C_{1}(s)=K_{c}(a s+1)
$$

Where $K_{c}$ is designed using the method described above so that the following equation has no RHP roots:

$$
T s-1+K \mathrm{e}^{-\theta s}=0
$$

where $K=K_{c} K_{p}$, so that

$$
\begin{gathered}
\hat{G}_{P}=\frac{K \mathrm{e}^{-\theta s}}{T s-1+K \mathrm{e}^{-\theta s}} \\
\hat{G}_{L}=\frac{K p \mathrm{e}^{-\theta s}}{(a s+1)\left(T-1+K \mathrm{e}^{-\theta s}\right)} G_{L}(s)
\end{gathered}
$$

According to $\hat{G}_{P}$

$$
\begin{gathered}
{\left[\hat{G}_{P}\right]_{-}=\frac{K}{T s-1+K \mathrm{e}^{-\theta s}}} \\
{\left[\hat{G}_{p}\right]_{+}=\mathrm{e}^{-\theta s}}
\end{gathered}
$$

The IMC controllers $q_{a}$ and $q_{b}$ (referring to Fig. 7) are synthesised by eqns. 17 and 18 as in the FODUP case. Thus, $G_{c}^{1}$ and $G_{c}^{2}$ are synthesised by eqns. 6 and 7 immediately.

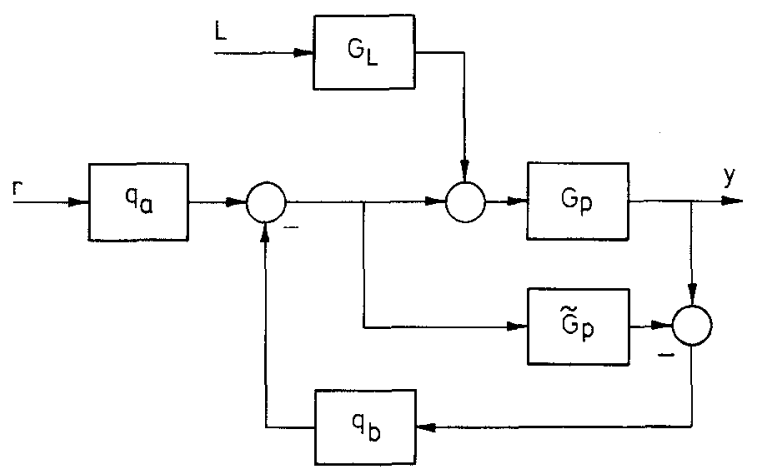

Fig.7 2DF IMC structure

It is obvious that, when the process is considered to be in the nominal case, the process output in the proposed method would be

$$
y=q_{a} \hat{G}_{p} r+\left(1-q_{b} \hat{G}_{p}\right) \hat{G}_{L} L
$$

Obviously, $q_{a}$ and $q_{b}$ are designed to improve the performance in reference tracking and disturbance rejection, respectively. Thus, the proposed method could allow the reference-tracking problem and the disturbance-rejection problem to be considered independently.

\section{Design of PID control system}

There are two different forms of the ideal PID controller: the parallel form $G_{c}(s)=K_{c}\left\{1+\left(1 / \tau_{R} s\right)+\tau_{D} s\right\}$ and the series form $G_{c}(s)=K_{c}\left\{1+\left(1 / \tau_{R} s\right)\right\}\left(1+\tau_{D} s\right)$. Although the parallel-form expression is more common in the literature, the series-form expression is adopted in this work for convenience in formulation. The methods of parameters interchange between these two forms are well known and can easily be found in many control textbooks $[1,24]$.

In the following, a tuning method is developed for a PID controller from the previous three-elementstructure design. Obviously, the series-form PID con- trol system has only one degree of freedom in design, so it is not directly compatible with a three-element system without simplification. However, as is discussed below, if some further assumptions are made, the proposed three-element design can be approximated to a PID one.

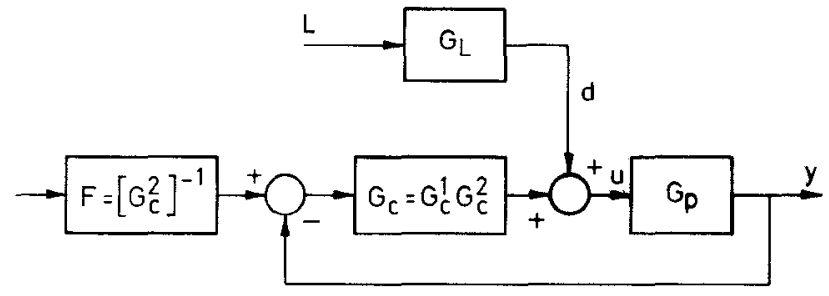

Fig. 8 2DF structure II

Referring to Fig. 4, if controller $G_{c}^{2}$ is moved from the feedback path to the forward path, the whole system will turn into that shown in Fig. 8. In Fig. 8, the main controller in the loop should be

$$
G_{c}=C_{1}\left(1+C_{2} C_{3}\right)=C_{1}\left(1+\frac{q_{b}}{1-q_{b} \hat{G}_{p}}\right)
$$

and the prefilter for the setpoint input is

$$
\hat{F}=\frac{C_{2}}{1+C_{2} C_{3}}
$$

To simplify this $2 \mathrm{DF}$ control system to a $1 \mathrm{DF}$ PID one, further assumptions are necessary. Since, in a $1 \mathrm{DF}$ control system, there will be no extra degree of freedom to treat servotracking and disturbance rejection independently, the first simplification is to make the IMC filters $f_{a}$ and $f_{b}$ equal. Consequently, $C_{3}$ will equal unity. As a result, $F$ and $G_{c}$ will be equal to $C_{2} /\left(1+C_{2}\right)$ and $C_{1}\left(1+C_{2}\right)$, respectively. According to eqn. 19, $C_{2}$ approaches infinity in the low-frequency range so that $\hat{F}$ can be taken as unity therein. Then, if eqn. 28 is examined, it can be seen that $\hat{F}$ functions like a lowpass filter with unity gain in low-frequency range. Note that the main loop in Fig. 8 is stabilised by $G_{c}$ and the main concern of control signals is emphasised in the low-frequency range. It is thus reasonable to take $\hat{F}$ as unity as the second assumption for simplification which would not endanger the stability and performance of the system. Because controller $C_{1}$ is indeed a PD controller, controller $G_{c}=C_{1}\left(1+C_{2}\right)$ can be approximated to a series-form PID controller as depicted in the following.

\section{Case (i): FODUP}

In this case,

$$
\begin{gathered}
C_{1}(s)=K_{c}(b s+1) \\
C_{2}(s)=\frac{T s-1+K(b s+1) e^{-\theta s}}{K(b s+1)\left\{\left(\tau_{f} s+1\right)^{N}-\mathrm{e}^{-\theta s}\right\}} \\
C_{3}(s)=1
\end{gathered}
$$

where $\tau_{f}$ and $N$ are the IMC filter constant and the degree of the filter, respectively. In this work, $N$ is chosen as 2 , and it can trivially be shown that $C_{2}(s)$ has the following properties according to this choice:

$$
\begin{gathered}
\lim _{s \rightarrow 0} C_{2}(s)=\infty \\
\lim _{s \rightarrow \infty} C_{2}(s)=0 \\
\lim _{s \rightarrow 0} \frac{1}{s C_{2}(s)}=\frac{K\left(2 \tau_{f}+\theta\right)}{K-1} \triangleq \tau_{R}
\end{gathered}
$$


From eqn. 34, $C_{2}(s)$ has just one integral mode. By making use of eqns. 32,33 and 34 , it is reasonable to approximate $1+C_{2}(s)$ with a PI controller. So $G_{c}(s)=$ $C_{1}(s)\left\{1+C_{2}(s)\right\} \simeq K_{c}(1+b s)\left\{1+\left(1 / \tau_{R} \mathrm{~s}\right)\right\}$ is approximated to a series-form PID controller.

Case (ii): SODUP

In this case,

$$
\begin{gathered}
C_{1}(s)=K_{c}(a s+1) \\
C_{2}(s)=\frac{T s-1+K \mathrm{e}^{-\theta s}}{K\left\{\left(\tau_{f} s+1\right)^{N}-\mathrm{e}^{-\theta s}\right\}} \\
C_{3}(s)=1
\end{gathered}
$$

where $\tau_{f}$ and $N$ are the IMC filter constant and the degree of the filter, respectively. In this work, $N$ is chosen as 2, and it can trivially be shown that $C_{2}(s)$ has the following properties according to this choice:

$$
\begin{gathered}
\lim _{s \rightarrow 0} C_{2}(s)=\infty \\
\lim _{s \rightarrow \infty} C_{2}(s)=0 \\
\lim _{s \rightarrow 0} \frac{1}{s C_{2}(s)}=\frac{K\left(2 \tau_{f}+\theta\right)}{K-1} \triangleq \tau_{R}
\end{gathered}
$$

For similar reasons to the FODUP case, $G_{c}(s)=$ $C_{1}(s)\left\{1+C_{2}(s)\right\}$ can be approximated to a PID controller.

The above discussions are summarised in Table 1.

Table 1: PID controller settings for FODUP and SODUP

\begin{tabular}{lll}
\hline $\mathrm{Gp}(\mathrm{s})$ & $\tau_{R}$ & $\tau_{D}$ \\
\hline$K_{p} \mathrm{e}^{-\theta s} / T s-1$ & $K\left(2 \tau_{f}+\theta\right) / \mathrm{K}-1$ & $\mathrm{~b}$ \\
$K_{p} \mathrm{e}^{-\theta s} /(T s-1)(a s+1)$ & $K\left(2 \tau_{f}+\theta\right) / \mathrm{K}-1$ & $\mathrm{a}$ \\
\hline
\end{tabular}

Note: $K=K_{c} K_{p}$

\section{Simulation examples}

In this Section, three examples are simulated to check the feasibility of the proposed method. The first example is a typical FODUP for which $\theta / T$ is smaller than unity. Some results in the literature for such an example are also included to compare with the proposed method. The second one is a FODUP for which $\theta / T$ is larger than unity. The third is an example of a highorder process. It is approximated to a SODUP first, and then the control system is synthesised according to this SODUP model. In all the examples, simulations in both the three-element system and PID control system are included. The integration interval is taken to be 0.005 in all simulation examples.

\section{Example 1}

Consider an unstable plant as:

$$
G_{p}(s)=\frac{\mathrm{e}^{-0.4 s}}{s-1}
$$

Referring to Fig. 6, the optimum value of $\hat{b}$ is 0.121 corresponding to $\theta / T=0.4$; thus the stabilising interval $\left(K_{m}, K_{M}\right)$ is $(1,4.17)$. The stabilising controller is then chosen as $C_{1}(s)=2.58(0.121 s+1)$. IMC filters are chosen as $f_{a}=1 /(s+1)$ and $f_{b}=1 /(0.2 s+1)$, so the IMC controllers are

$$
\begin{aligned}
& q_{a}=\frac{s-1+2.58(0.121 s+1) \mathrm{e}^{-0.4 s}}{2.58(0.121 s+1)(s+1)} \\
& q_{b}=\frac{s-1+2.58(0.121 s+1) \mathrm{e}^{-0.4 s}}{2.58(0.121 s+1)(0.2 s+1)}
\end{aligned}
$$

The simulation results for setpoint tracking and disturbance rejection are shown in Figs. 9 and 10. When the three-element system is simplified into the PID control system, a more conservative value of IMC filter is chosen to assure robust stability. In this example, the filter constant is chosen to be 1.5 , and the corresponding tuning parameters in a series PID controller are then calculated as: $K_{c}=2.58, \tau_{R}=5.552$ and $\tau_{D}=0.121$.

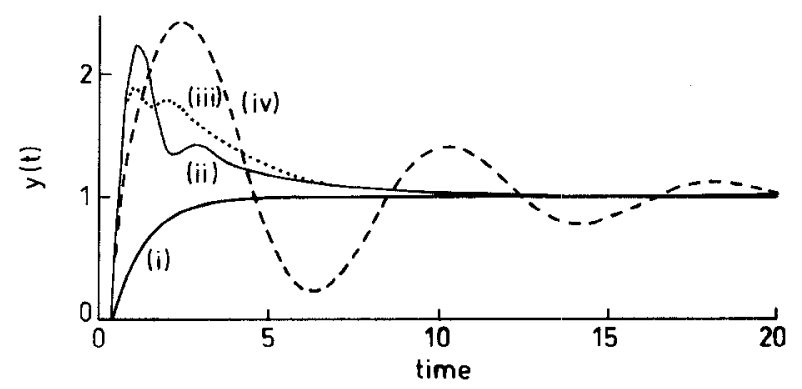

Fig.9 Nominal responses of different methods for example 1: setpoint tracking

(i) proposed three-element

(ii) proposed PID

(iii) Rotstein et al.

(iv) De Paor et al.

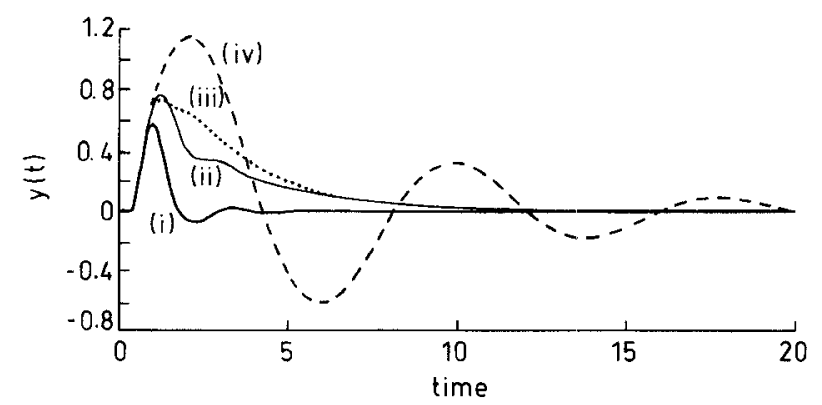

Fig. 10 Nominal responses of different methods for example 1: disturbance attenuation

(i) proposed three-element

(ii) proposed PID

(iv) De Paor et a1.

For the existing methods in the literature, Shafiei and Sehenton's work needs to assign the closed-loop pole of the control system first, but does not address the method of choosing the proper pole [10]. Venkatashankar and Chidambarams' work proposed a method to tune only the PI controller, and it is obvious that its performance is inferior to that of a PID one [11]. Huang and Lins' method is indeed a 2DF PIDcontroller-tuning method, and the controller output of such a method is modified by a nonlinear saturation element, but it does not address how to determine the saturation range [12]. Therefore the above-mentioned studies are not compared with the proposed method. However, the work of De Paor and O'Malley [8] and Rotstein and Lewin [9] is compared with the proposed method in this example.

Tuning parameters in the PID controller of this example for these two studies and the proposed method are listed in Table 2. Note that the PID-parameter list in Table 2 is based on the parallel-form PID controller.

Table 2: Different PID-controller tuning methods for example 1

\begin{tabular}{lllll}
\hline & Form & $K_{o}$ & $\tau_{R}$ & $\tau_{D}$ \\
\hline Depaor and O'Malley & Parallel & 1.459 & 2.667 & 0.250 \\
Rotstein and Lewin & Parallel & 2.250 & 5.760 & 0.200 \\
This work & Parallel & 2.636 & 5.673 & 0.118 \\
\hline
\end{tabular}


As shown in Figs. 9 and 10, the overshoot of all the PID-tuning methods is over $100 \%$. However, this fact can be understood from theorem 2. The minimum overshoot of any feedback controller will be $\mathrm{e}^{0.4}-1=$ 0.492, if perfect control is possible. Because the PID controller is a low-order controller with a specific structure, the real overshoot in the control system would be much higher than that minimum value. In our experience, the overshoot would be about 2-4 times this minimum value in a PID control system. In the three-element structure, the overshoot is, indeed, suppressed, as shown in the simulation results.

For comparison, the integral absolute error (IAE) corresponding to all the methods for this example are listed in Table 3.

Table 3: IAE of example 1 for different methods

\begin{tabular}{lll}
\hline & Tracking & Attenuation \\
\hline Depaor and O'Malley & 7.855 & 5.789 \\
Rotstein and Lewin & 3.553 & 2.559 \\
Proposed PID & 3.177 & 2.148 \\
Proposed three-element & 1.445 & 0.515 \\
\hline
\end{tabular}

As Table 3 shows, the proposed three-element method improves the performance in both tracking and attenuation considerably according the IAE performance index. The proposed PID method is also superior to the other two methods in the sense of IAE. Although there seem to be no significant improvements in setpoint tracking when the proposed PID method is compared with Rotstein and Lewins' method, the proposed method behaves better than their method in the attenuation case. Moreover, note that the method suggested by Rotstein and Lewin is limited to $\theta / T<1$ for a FODUP but the proposed method can be applied to the case of $\theta / T<2$.

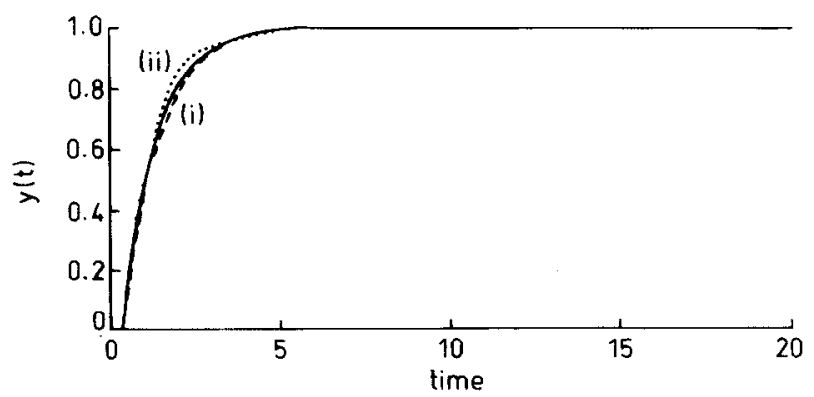

Fig. 11 Robust response of three-element systems for example 1: perturbation in $K_{n}$

nominal case

(i) positive perturbation

(ii) negative perturbation

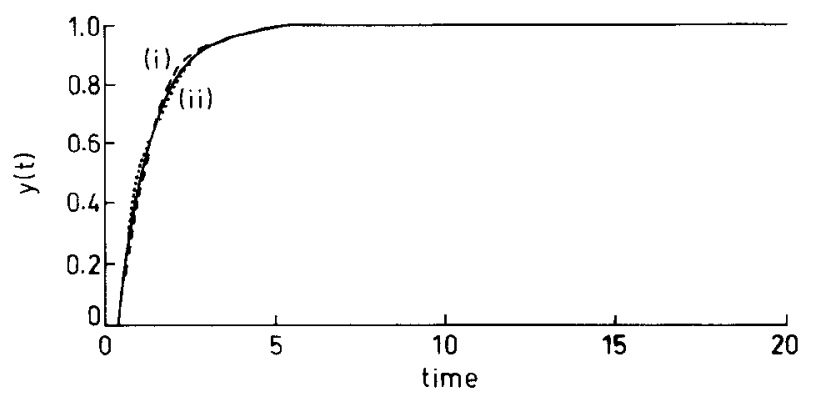

Fig.12 Robust response of three-element systems for example 1: periurbation in $T$

(i) positivinal case

(i) positive perturbation

(ii) negative perturbation

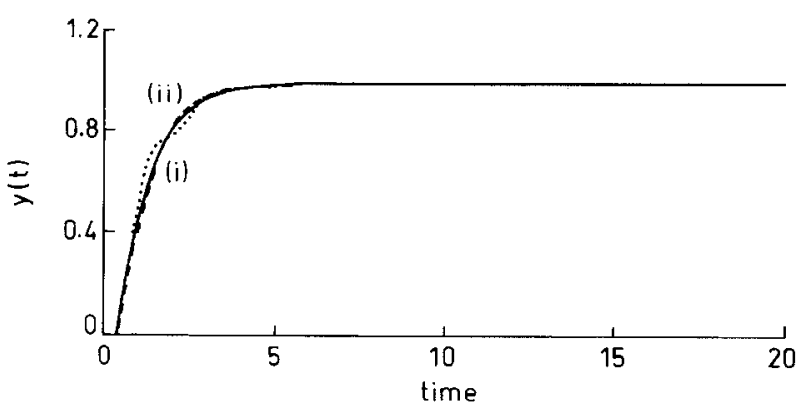

Fig. 13 Robust response of three-element systems for example 1: perturbation in $\theta$

nominal case

(i) positive perturbation

(ii) negative perturbation

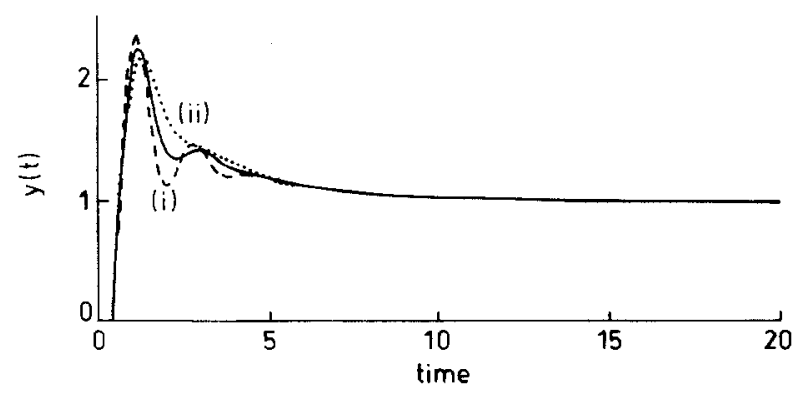

Fig.14 Robust response of PID control system for example 1: perturbation in $K_{p}$

(i) positive perturbat

(ii) negative pertur

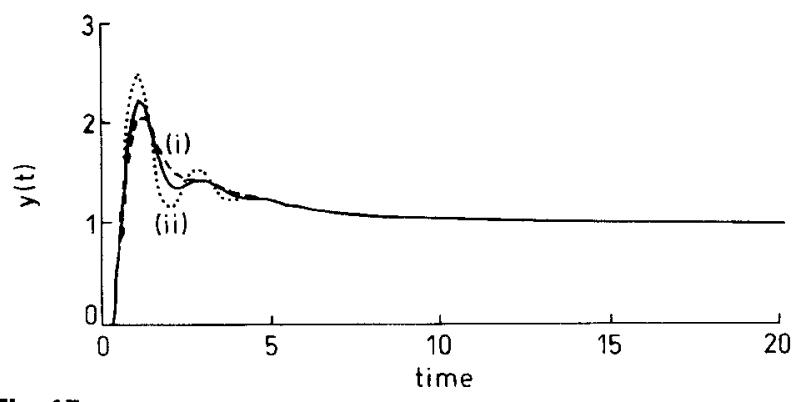

Fig.15 Robust response of PID control system for example 1: perturbation in $T$

(i) pominal case

(i) positive perturbation

(ii) negative perturbation

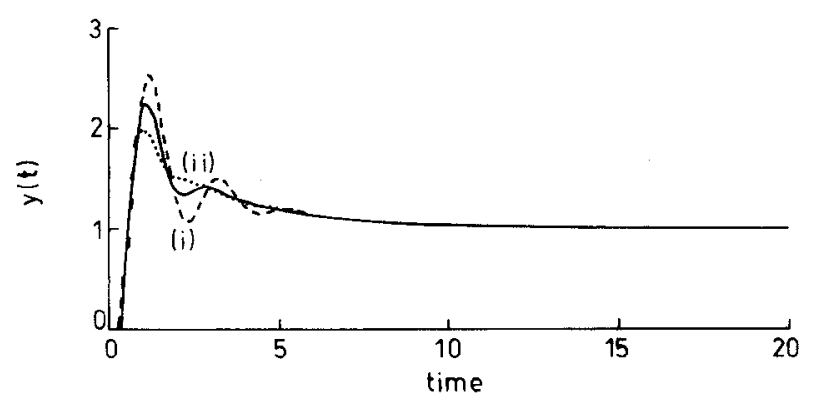

Fig.16 Robust response of PID control system for example 1: perturbation in $\theta$

(i) positive perturbation

(ii) negative pertur

It is assumed that there are $\pm 10 \%$ parameter perturbations in $K_{p}, T$, and $\theta$, respectively, and the foregoing design parameters are then applied to test the robustness of the proposed methods. As shown in Figs. 1116 , the proposed methods are robust to parameter perturbation in this example. 


\section{Example 2}

Consider an unstable plant as

$$
G_{p}(s)=\frac{\mathrm{e}^{-1.2 s}}{s-1}
$$

Referring to Fig. 6, the optimum value of $\hat{b}$ is 0.524 corresponding to $\theta / T=1.2$; thus the stabilising interval $\left(K_{m}, K_{M}\right)$ is $(1,1.32)$. The stabilising controller is then chosen as $C_{1}(s)=1.16(0.524 s+1)$. The IMC filters are chosen as $f_{a}=1 /(3 s+1)$ and $f_{b}=1 /(1.5 s+1)$, respectively. So, the IMC controllers would be

$$
\begin{aligned}
q_{a} & =\frac{s-1+1.16(0.524 s+1) \mathrm{e}^{-1.2 s}}{1.16(0.524 s+1)(3 s+1)} \\
q_{b} & =\frac{s-1+1.16(0.524 s+1) \mathrm{e}^{-1.2 s}}{1.16(0.524 s+1)(1.5 s+1)}
\end{aligned}
$$

The simulation results for setpoint tracking and disturbance rejection are shown in Figs. 17 and 18. When the three-element system is simplified into the PID control system, the filter constant is chosen to be 3.0 , and the corresponding tuning parameters in a series PID controller are then calculated as $K_{c}=1.16, \tau_{R}=52.20$ and $\tau_{D}=0.524$. To the authors' knowledge, PID-controllerdesign methods in the literature do not apply to this example [8-12].

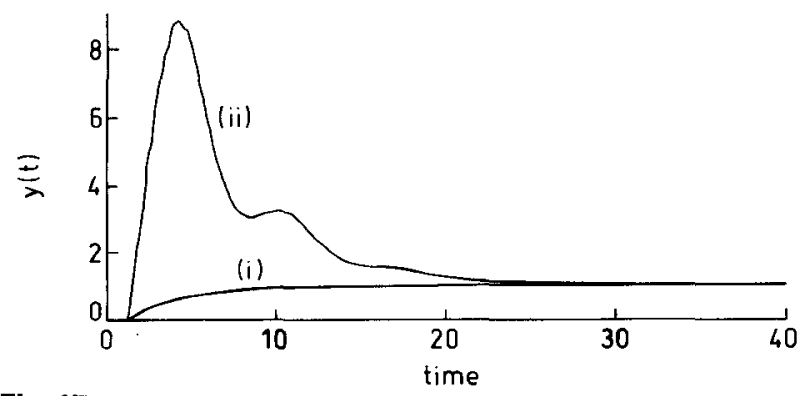

Fig. 17 Nominal responses of different methods for example 2: setpoint tracking

(i) proposed three-element

(ii) proposed PID

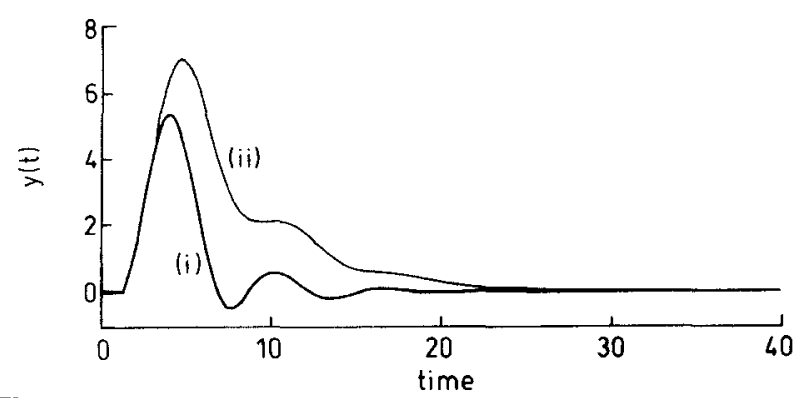

Fig. 18 Nominal responses of different methods for example 2: disturbance rejection

(i) proposed three-element

(ii) proposed PID

In tuning a PID controller for a stable process, it makes no difference whether the controller parameters are tuned in the sequence $K_{c}, \tau_{R}$ and $\tau_{D}$, or in the sequence $K_{c}, \tau_{D}$ and $\tau_{R}$. However, this is not true for tuning of a PID controller for an unstable process. In the proposed method, the PID controller is tuned in the sequence of $K_{c}, \tau_{D}$ and $\tau_{R}$. This is why the proposed PID method still works for $1 \leq \theta / T<2$.

For the purpose of comparison, the integral absolute error (IAE) corresponding to the three-element system and the PID system for this example are listed in Table 4 .

Table 4: IAE of example 2 for different methods

\begin{tabular}{lll}
\hline & Tracking & Attenuation \\
\hline Proposed PID & 47.65 & 44.98 \\
Proposed three-element & 4.29 & 18.72 \\
\hline
\end{tabular}

As Table 4 shows, the proposed threemelement method improves the performance in both tracking and attenuation considerably, according the IAE performance index. To test the robustness-performance issues of the proposed methods, it is assumed that there are $\pm 5 \%$ parameter perturbations in $K_{p}, T$ and $\theta$, respectively. The foregoing design parameters are then introduced to test the robustness performance of the proposed methods. As shown in Figs. 19-24, the proposed methods are robust to parameter perturbation in this example.

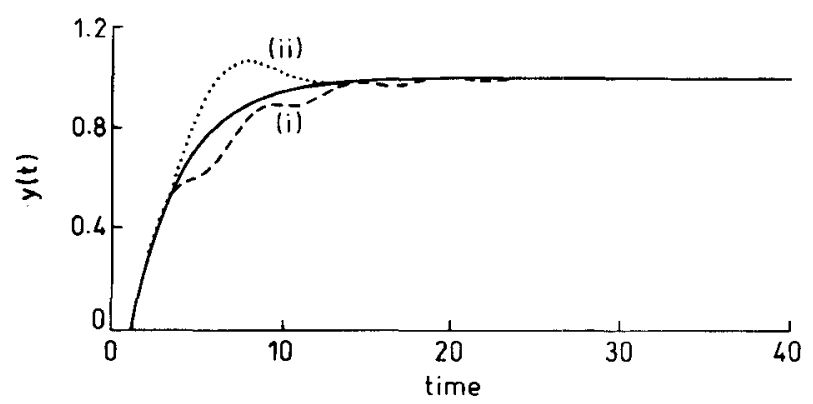

Fig. 19 Robust response of three-element system for example 2: perturbation in $K_{p}$ nominal case (i) positive perturbation (ii) negative perturbation

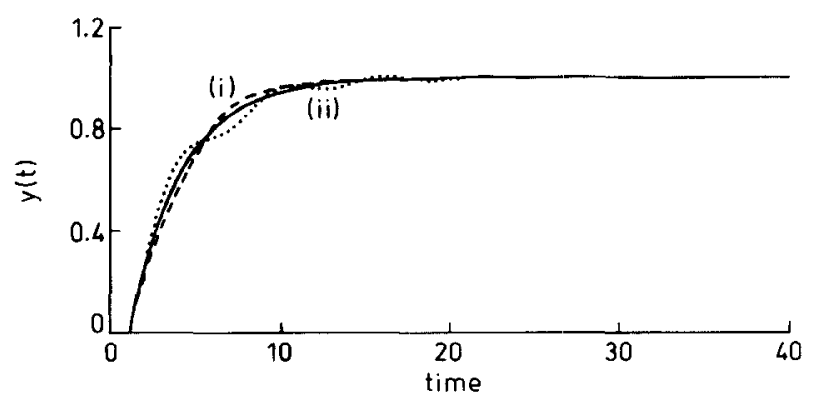

Fig. 20 Robust response of three-element system for example 2: perturbation in $T$ - nominal case

(i) positive perturbation

(ii) negative perturbation

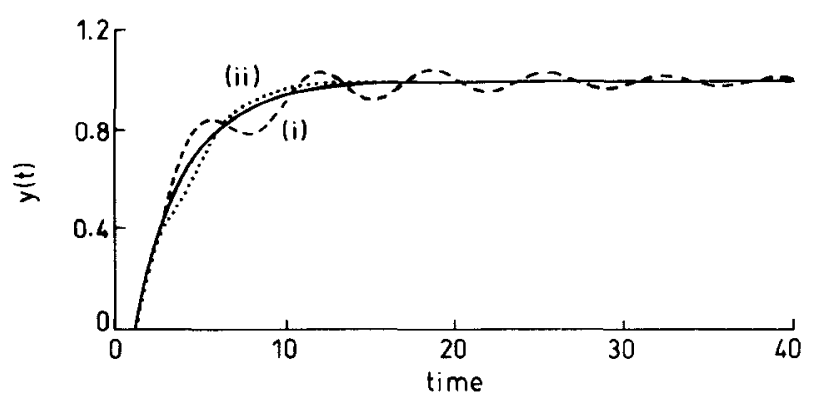

Fig.21 Robust response of three-element system for example 2: perturbation in $\theta$

nominal case

(i) positive perturbation

(ii) negative perturbation 


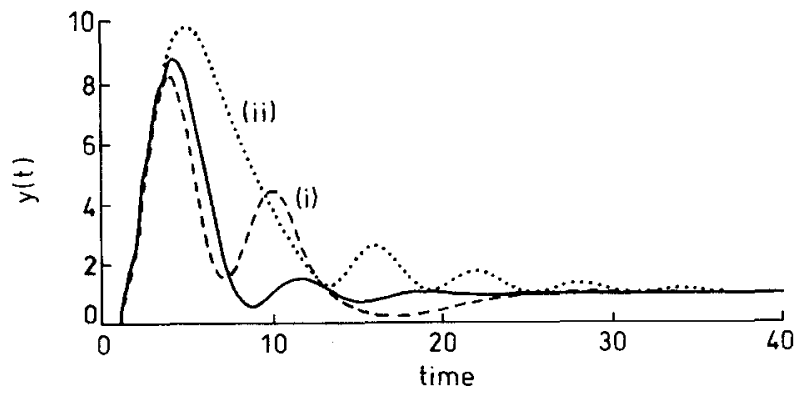

Fig.22 Robust response of PID control system for example 2: perturbation in $K_{p}$

(i) pominal case

positive perturbation

(ii) negative perturbation

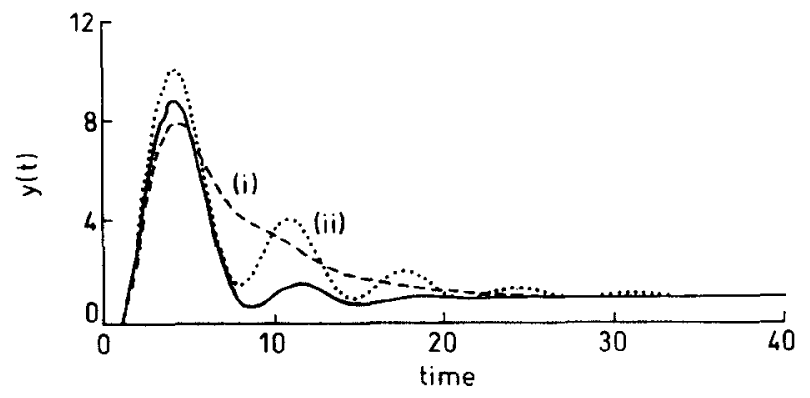

Fig.23 Robust response of PID control system for example 2: perturbation in $T$

$\longrightarrow$ nominal case

(i) positive perturbation

(ii) negative perturbation

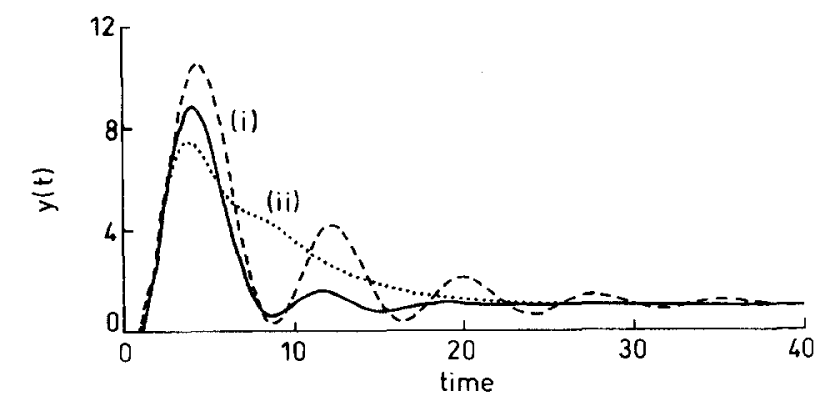

Fig.24 Robust response of PID control system for example 2: perturbation in $\theta$

(i) nositive nertul case

(i) positive perturbation

(ii) negative perturbation

\section{Example 3}

Consider an unstable plant as

$$
G_{p}(s)=\frac{e^{-0.5 s}}{(5 s-1)(2 s+1)(0.5 s+1)}
$$

It is well known that the stable, higher-order part of a transfer function can be approximated effectively to a first-order delayed transfer function. Let us approximate this transfer function as [12]

$$
G_{p}(s) \simeq \frac{e^{-0.939 s}}{(5 s-1)(2.07 s+1)}
$$

Thus the stabilising controller $C_{1}(s)$ is synthesised as $C_{1}(s)=4.4(2.07 s+1)$. IMC filters are chosen to be $f_{a}=$ $1 /(2 s+1)$ and $f_{b}=1 /(1.5 s+1)$, respectively. The IMC controllers are then derived as

$$
\begin{aligned}
q_{a} & =\frac{5 s-1+4.4 \mathrm{e}^{-0.939 s}}{4.4(2 s+1)} \\
q_{b} & =\frac{5 s-1+4.4 \mathrm{e}^{-0.939 s}}{4.4(1.5 s+1)}
\end{aligned}
$$

The settings of the series PID controller are $K_{c}=4.4$, $\tau_{D}=2.07$ and $\tau_{R}=5.10$ if the IMC filter constant is chosen to be 1.5. For comparison, the integral absolute error (IAE) corresponding to the three-element system and the PID system for this example (Figs. 25 and 26) are listed in Table 5.

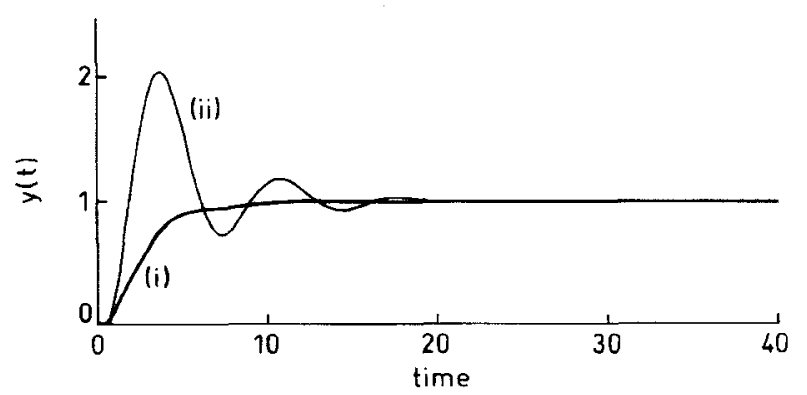

Fig.25 Nominal responses of different methods for example 3: setpoint tracking

(i) proposed three-element

(ii) proposed PID

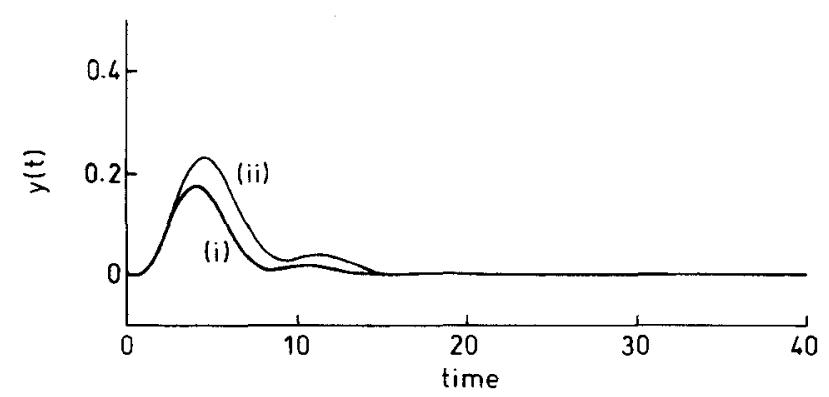

Fig.26 Nominal responses of different methods for example 3: disturbance rejection

(i) proposed three-element

(ii) proposed PID

Table 5: IAE of example 3 for different methods

\begin{tabular}{lll}
\hline & Tracking & Attenuation \\
\hline Proposed PID & 1.16 & 5.37 \\
Proposed three-element & 0.72 & 3.04 \\
\hline
\end{tabular}

As Table 5 shows, the proposed three-element method improves the performance in both tracking and attenuation significantly, according the IAE performance index. To test the robustness-performance issues of the proposed methods, it is assumed that there are $\pm 20 \%$ parameter perturbations in $K_{p}, T$ and $\theta$, respectively. As shown in Figs. 27-32, the proposed methods are robust to parameter perturbation in this example.

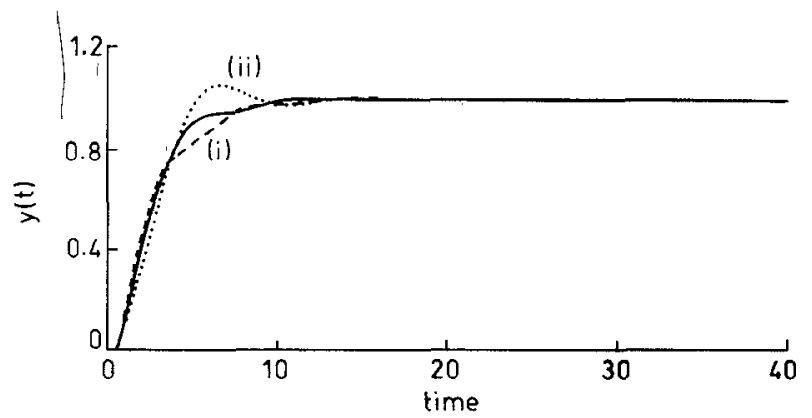

Fig.27 Robust response of three-element system for example 3: perturbation in $K_{p}$

(i) positive perturbation

(ii) negative perturbation 


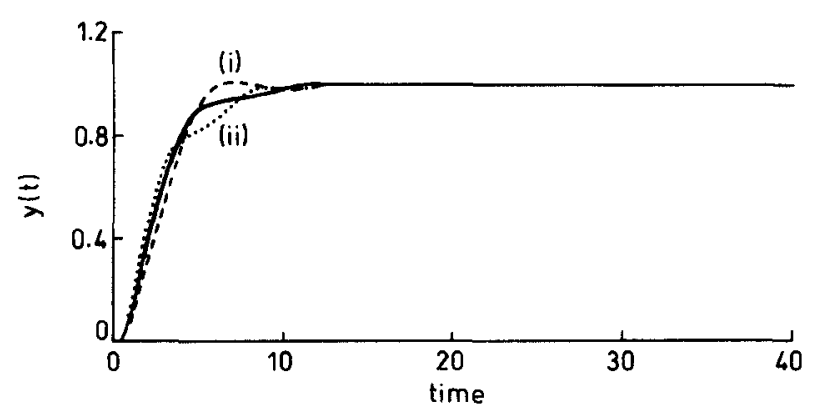

Fig.28 Robust response of three-element system for example 3: perturbation in $T$

(i) positive perturba

(ii) negative

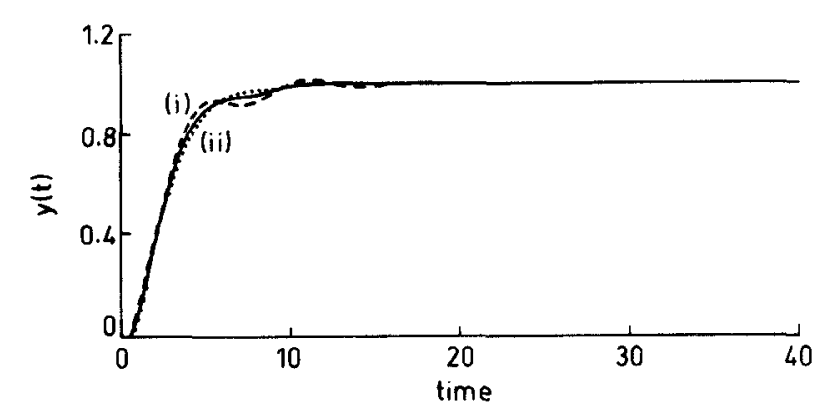

Fig. 29 Robust response of three-element system for example 3: perturbation in $\theta$

(i) nominal case

(i) positive perturbation

(ii) negative perturbation

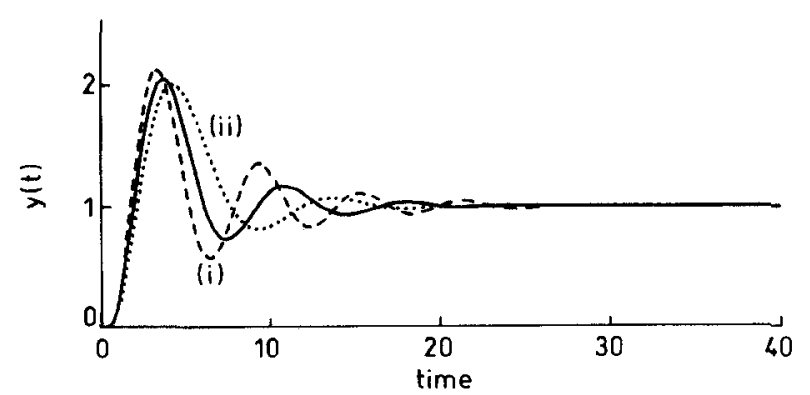

Fig.30 Robust response of PID control system for example 3: perturbation in $K_{p}$

nominal case

(i) positive perturbation

(ii) negative perturbation

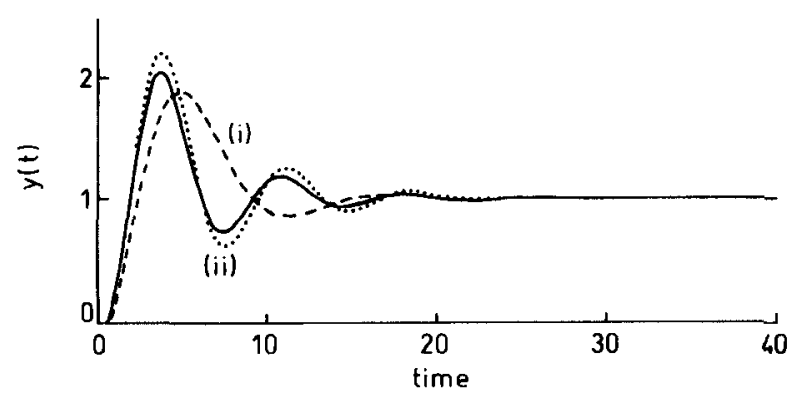

Fig.31 Robust response of PID control system for example 3: perturbation in $T$

nominal case

(i) positive perturbation

(ii) negative perturbation

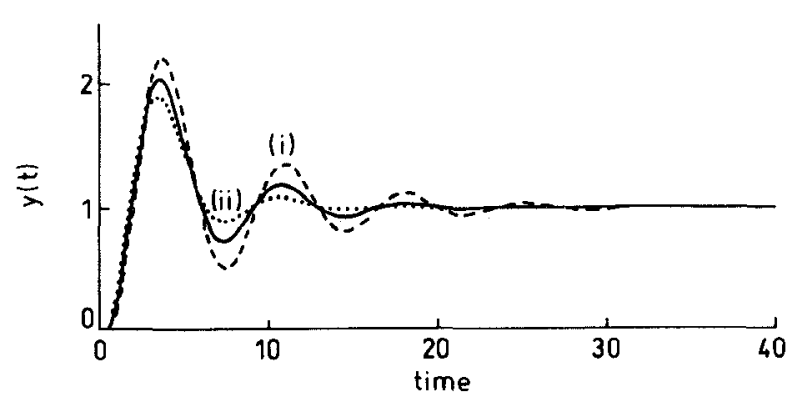

Fig.32 Robust response of PID control system for example 3: perturbation in $\theta$

nominal case

(i) positive perturbation

(ii) negative perturbation

\section{Conclusions and discussion}

Open-loop unstable processes are considered to be difficult to control in processes industries. In this paper, performance degradations for unstable processes are studied based on both the time domain and the frequency domain to highlight these difficulties which are imposed on the overshoot and the infinity norm of the complementary sensitivity function. To avoid these difficulties, a three-element structure, which is indeed a $2 \mathrm{DF}$ structure, is proposed to handle this problem effectively. A PID tuning method evolved from the proposed structure is also suggested. Simulation examples show a reasonable performance and robustness of the proposed method. Thus, the proposed method provide a simple and effective controller-design technique for the time-delayed unstable process.

Although the performance of the proposed design method in the three-element structure is much better than that in the traditional unity-feedback structure in both tracking and attenuation, according to IAE performance index, as shown in the simulation examples, the improvement in disturbance attenuation is still not very satisfactory. To make up this deficiency, a new type of the IMC filter for $q_{b}$ should be considered, instead of the standard IMC filter. This is a topic for future study.

The strongly stabilising conditions for the timedelayed unstable process are still unclear in the literature. This means that we do not know when we must use an unstable controller to stabilise a time-delayed unstable process. Thus, the proposed work is indeed confined to the process which can be stabilised by a $\mathrm{PD} / \mathrm{PID}$ controller only. The strongly stabilising problem for the time-delayed unstable process is another challenging topic for the future.

\section{References}

1 KUO, B.C.: 'Automatic control systems' (Prentice-Hall, 1991, 6th edn.)

2 VIDYASAGAR, M.: 'Control system synthesis-a factorization approach' (MIT Press, 1985)

3 YOULA, D.C., BONGIORNO, J.J.J., and JABR, H.A.: 'Modern Wiener-Hoppf design of optimal controllers - part I: The single-input-output case', IEEE Trans., 1976, AC-21, pp. 3-13

4 FREUDENBERG, J.S., and LOOZE, D.P.: 'Right half plane poles and zeros and design tradeoffs in feedback system', IEEE Trans., 1985, AC-30, pp. 555-565

5 FREUDENBERG, J.S., and LOOZE, D.P.: 'A sensitivity tradeoff for plants with time delay', IEEE Trans., 1987, AC-32, pp. 99-104

6 LOOZE, D.P., and FREUDENBERG, J.S.: 'Limitations of feedback properties imposed by open-loop right half plane poles', IEEE Trans., 1991, AC-36, pp. 736-739

7 DOYLE, J.C., FRANCIS, B.A., and TANNENBAUM, A.R 'Feedback control theory' (Macmillan Publishing Co., 1992) 
8 DE PAOR, A.M., and O'MALLEY, M.: 'Controller of zieglernichols type for unstable process with time delay', Int. J. Control, 1989, 49, pp. 1273-1284

9 ROTSTEN, G.E., and LEWIN, D.R.: 'Simple PI and PID tuning for open-loop unstable systems', I\&EC, 1991, 30, pp. 1864 1869

10 SHAFIEI, Z., and SHENTON, T.: 'Tuning of PID-type controller for stable and unstable systems with time delay', Automatica, 1994, 30, pp. 1609-1615

11 VENKATAHANKAR, $V$., and CHIDAMBARAM, M: 'Design $\mathrm{P}$ and PI controller for unstable first-order plus time delay process', Int. J. Control, 1994, 60, pp. 137-144

12 HUANG, C.T., and LIN, Y.S.: 'Tuning PID controller for openloop unstable process with time delay', Chem. Eng. Comm., 1995, 33, pp. $11-30$

13 CHIDAMBARAM, M.: 'Design of PI and PID controllers for an unstable first-order plus time delay system', Hung. J. Ind. Chem., 1995, 23, pp. 123-127

14 LUYBEN, W.L.: 'Process modelling simulation and control for chemical engineers' (McGraw-Hill, 1990, 2nd edn.)

15 LUYBEN, W.L.: "Simple method for tuning siso controllers in multivariable systems', Ind. Eng. Chem, Process Des. Dev., 1986, 25, pp. 654-660
16 SANATHANAN, C.K., and QUINN, J.S.B.: "Controller design, for integrating and runaway processes involving time delay', AIChE J., 1989, 35, pp. 923-930

17 MORARI, M., and ZAFIRIOU, E.: 'Robust process control' (Prentice-Hall, 1989)

18 JACOB, E.F., and CHIDAMBARAM, M.: 'Design of controllers for unstable first-order plus time delay model', Comput. Chem. Eng., 1996, 20, (5), pp. 579-584

19 CHEN, C.T.: "Control system design - conventional, algebraic and optimal method' (Pond Woods, 1987)

20 CAMPI, M., LEE, W.S., and ANDERSON, B.D.O.: 'Filters for internal model control design for unstable plants'. Proceedings of the $32 \mathrm{nd}$ conference on Decision and control, San Antonio, Texas, 1993, pp. $1343-1348$

21 DE PAOR, A.M.: 'A modified smith predictor and controller for unstable process with time delay', Int. $J$. Control, 1985,41 , pp. $1025-1036$

22 HUANG, H.P., and CHEN, C.C.: 'On stabilising a time delayed unstable process', Chin. I. Ch. E., submitted for publication

23 HUANG, H.P., and CHEN, C.C.: "Control system design for openloop unstable process having time delay - a 3-block openloop, unstable process having time delay - a a 3-block July 1996 , pp. 619-624

24 ASTROM, K.J., and HAGGLUND, T.: 'PID controllers' (Instument Society of America, 1995) 